\title{
Development of a magnetic targeting device applied to interlocking of distal intramedullary nail screw holes.
}

\author{
by \\ David C. Szakelyhidi Jr. \\ Thesis submitted to the Faculty of Virginia Polytechnic Institute and State University \\ in partial fulfillment of the requirements for the degree of \\ Master of Science \\ In \\ Mechanical Engineering
}

Approved:

Dr. A. Wicks, Chair

Dr. E. Scott

Dr. A. Durham

May, 2002

Blacksburg, Virginia

Keywords: Intramedullary Nailing, Distal Interlocking, Magnetic Targeting 


\title{
Development of a magnetic targeting device applied to interlocking of distal intramedullary nail screw holes.
}

\author{
David C. Szakelyhidi Jr.
}

Committee Chair: Dr. A. Wicks

\author{
Abstract \\ Mechanical and Biomedical Engineering
}

\begin{abstract}
Each year, thousands of femoral and tibial internal fracture repairs are performed by orthopedic surgeons in the United States. Internal fixation of long bones using intramedullary nails (IMN) has decreased incidence of non-union, allowed shorter hospitalization time, and earlier weight bearing for the patient compared to other fixation methods. Orthopedic surgeons have expressed that one of the most difficult parts of this intramedullary nailing of long bones, is locating and drilling the interlocking screw holes. IMN interlocking requires the surgeon to locate the holes in the nail, center the drill, and advance the bit through the bone to meet them.

Many novel procedures and devices have been developed to assist the surgeon in distal locking of intramedullary nails, but have some disadvantages. These can include the need for extensive x-ray exposure, expensive x-ray equipment, high power consumption, active electronics in vivo, soft tissue damage, which all lead to inaccurate screw placement. For these reasons, a new prototype device for locating and drilling IMN distal interlocking holes has been developed. This prototype device uses magnetic sensors to locate a permanent magnet placed at a know distance from the IMN interlocking hole. A drill sleeve may be attached to the targeting sensors so that when they are aligned with the target magnet, the drill sleeve is aligned with the axis of the interlocking hole to be drilled. This new prototype device has significant advantages over existing devices, including the following. It has no active or passive electronics in vivo, no x-ray imaging is needed for targeting, while allowing real time feedback of alignment. It is a percutaneous technique, which can be adapted for use with existing IMN's. The new prototype also has low power requirements allowing battery operation, a single target magnet with unique axisymmetric field and novel magnet orientation, and adjustable sensitivity. Additionally, the new device allows visual, audible, or tactile positioning feedback. This prototype magnetic targeting device has the potential to improve orthopedic surgeons' ability to target and drill distal IMN interlocking screw holes. This device may allow shorter surgery, decreased x-ray exposure, and fewer complications for the surgeon and patient.
\end{abstract}




\section{Acknowledgements}

I would like to thank all those who have supported my ever evolving research and those who have contributed to my education as an engineer, a researcher, and as a person. I thank the Carilion Biomedical Institute for their generous funding of my research and the opportunity to collaborate with such a knowledgeable group of physicians. I thank Dr. Wicks for teaching me the importance of hard work and balance, of which I am greatly appreciative. I would like to thank Dr. Durham for his contribution as a medical advisor to my research and general medical knowledge. I thank Dr. Scott for serving on my committee. I also thank Dr. Nunnally for taking the time to teach me the programming skills I needed to complete my projects. Lastly, I give special thanks to my parents, grandparents, brothers, sister, and friends who stuck it out with me while I made my way through these years of graduate school. Thank you. 


\section{Table of Contents}

Abstract__ ii

Acknowledgments__ iii

List of Figures___ V

Section 1, Introduction

Section 2, Motivation 22

Section 3, Approach _ 6

Section 4, Magnetic Field Targeting ___ 8

a) Target Magnet Selection___ 8

b) Targeting Sensors ___ 11

c) Targeting Array ___ 11

d) Positioning Feedback 12

Section 5, Prototype ___ 14

Section 6, Discussion _ 16

Section 7, Conclusions _ 17

References__ 18

Vita_ 20 


\section{List of Figures}

Figure 1) Intramedullary nail and fluoroscopic images of its insertion. The distal interlocking screws can be seen in the bottom of the x-rays.

Figure 2) Coordinate system applied to the distal end of IMN, showing all possible degrees of deformation.

Figure 3) Maximum tolerances for screw insertion angle and translation.

Figure 4) Perceived distal interlocking screw hole targeting device. The correct axis of alignment between the drill sleeve, hole, sensors, and target magnet are shown as the dotted lines.

Figure 5) Plots of flux density in the $y-z$ and $x-z$ plane above the chosen cylindrical magnet. The peak is centered exactly over the magnet, parallel with the z-axis, and is detectable at the required targeting distance of $10 \mathrm{~cm}$.

Figure 6) Plot of flux field around the magnet in the x-y plane. There exists a major and minor axis to the elliptic field, which can be differentiated by the targeting sensors to determine rotation about the z-axis. The north pole is facing in the positive $\mathrm{z}$ direction.

Figure 7) A portion of the mapping of flux density using a Gauss meter at $10 \mathrm{~cm}$ above the target magnet in the $+\mathrm{z}$-direction. Notice the axisymmetric field, as the model predicts in Figure 6.

Figure 8) A Model of flux lines produced by the magnet. The elliptic array is shown, in which each sensor lies perpendicular to the flux field. This arrangement allows for equivalent voltage outputs from all the sensors when the array is centered in the flux field over the magnet.

Figure 9) Arrangement of sensors fixed in a plastic housing to create an ellipse of opposing sensors pairs.

Figure 10) LED display arranged on the board in the form of a "bull's eye" for indicating correct alignment.

Figure 11) Prototype sensor array and LED display boards are shown connected and ready for targeting. The attached wires lead to the external positioning and calibration circuits.

Figure 12) The sensors embedded in the handle are shown centered over the target magnet. 


\section{Introduction}

Each year, approximately 14,000 femoral and 12,000 tibial internal fracture repairs are performed by orthopedic surgeons in the United States. Internal fixation of long bones has decreased incidence of non-union, allowed shorter hospitalization time, and earlier weight bearing for the patient compared to plaster cast or external fixation. ${ }^{1,4}$ The procedure involves fixation of long bone fractures by insertion of a long, thin-walled, metallic nail into the medullary canal of the damaged bone. The surgeon introduces the implant by creating a hole in the proximal end of the bone that opens into the canal. The bone fragments are aligned and the device is passed through them, so as to create a means for internal support. The nail is prefabricated with holes in the proximal and distal ends, which are meant to accept interlocking screws. The addition of screws is essential to control rotation and translation of the bone fragments with respect to each other. It is necessary to align and drill through the bone to meet the proximal and distal interlocking screw holes of the nail. An x-ray image and actual picture of an IMN can be seen in Figure1.
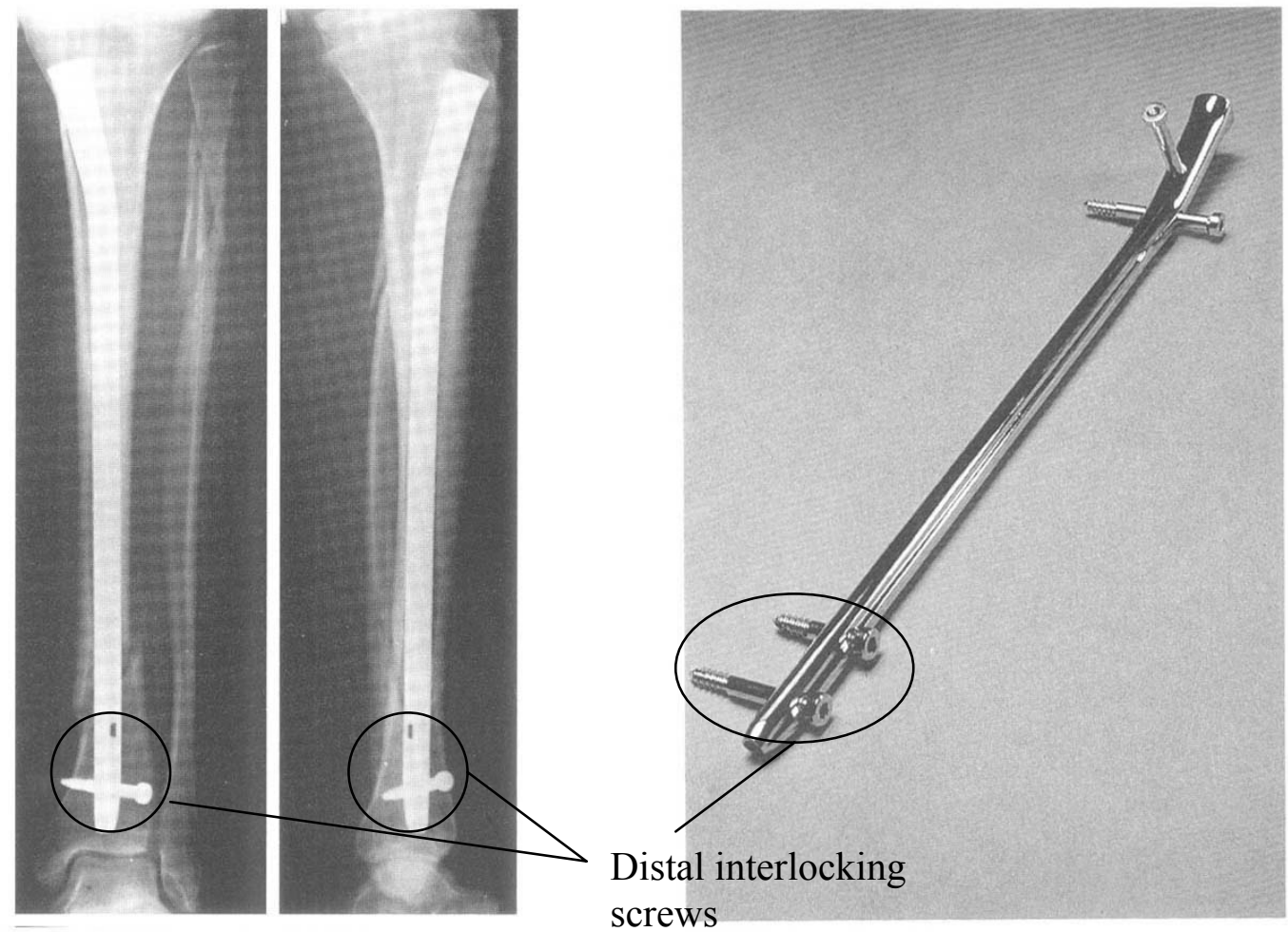

Figure 1) Intramedullary nail and fluoroscopic images of its insertion. The distal interlocking screws can be seen in the bottom of the x-rays. ${ }^{4}$

Since their introduction, all facets of the intramedullary nail (IMN) have evolved, from the shape and materials, to the methods and procedures used to implant them. While some procedures are generally agreed upon, orthopedic surgeons are in disagreement about many others. Discrepancies arise especially when looking at modern methods of interlocking screw insertion. In response, there is a great deal of work being done to improve methods for targeting, drilling, and inserting the distal interlocking 
screws. One important aspects of the IMN procedure is the placement of the interlocking screws. The performance of the surgeon and the device at this step can have a profound effect on the post-operative outcome. There is a wealth of literature available on the subject of intramedullary nailing of long bone fractures, but there is disagreement on which is the best device and procedure. One may be better suited for a certain fracture pattern than another. Improvements are needed for many of the devices. The first part of this research is to study current distal targeting techniques and find their advantages and disadvantages. From this review, a set of design requirements will be determined. Then, utilizing the design requirements, an alternative concept for interlocking screw hole targeting will be presented. The final efforts of this research will see the development of a prototype targeting device which can be applied to distal IMN interlocking screw hole locating and drilling.

\section{Motivation}

Orthopedic surgeons have suggested that one of the more difficult parts of intramedullary nailing of long bones, is locating and drilling the interlocking screw holes. ${ }^{10}$ IMN interlocking requires the surgeon to locate the holes in the nail, center the drill, and advance the bit through the bone to meet them. The interlocking screws are then inserted. Proximal interlocking screw placement is relatively easy because the holes can be located with an external guide attached to the end of the IMN. However, it is found that this technique does not work well for distal interlocking.

Studies conducted have found large values of medial-lateral and anterior-posterior flexion of the distal nail after it has been inserted..$^{10,11,13}$ On insertion, the distal IMN exhibited mean lateral deflections of $4.5 \pm 3.5 \mathrm{~mm}$ and dorsal deflections of $7.8 \pm 5.8 \mathrm{~mm}$. In addition, rotational deformations of the distal IMN have been measured at $0.3 \pm 0.7$ degrees. ${ }^{10}$ The orientation of coordinates is seen in Figure 2, where $\gamma, \beta$, and $\alpha$ indicate rotation about the $\mathrm{x}, \mathrm{y}$, and, $\mathrm{z}$ axis, respectively. The distal nail may deform from its original shape in any of these coordinates to some degree. The deformation of the nail is due to the shaping of the medullary canal where it is inserted. There are wide variations of the medullary canal shapes from person to person, and it is not currently possible to predict how the nail will deform accordingly. Therefore it is difficult to determine what the resultant location of the distal interlocking holes will be relative to their initial position.

There are tight tolerances between the screw and interlocking holes as seen in Figure 3. The angular deviation of the screw must be less than approximately eight degrees for no interference when inserted. Additionally, the lateral deviation should be less than 0.75 millimeter. To avoid any complications, it is important to place the interlocking screws accurately. These tolerances must be taken into account when targeting and drilling, so as to allow room for proper screw insertion. 


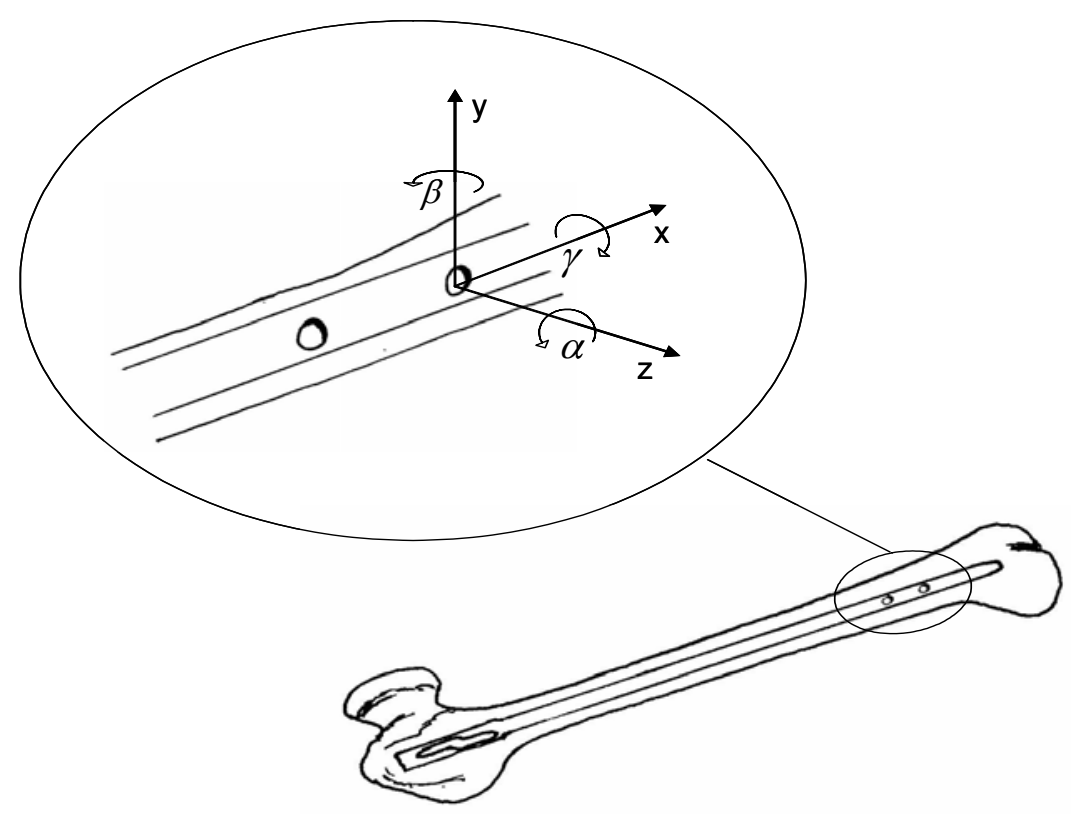

Figure 2) Coordinate system applied to the distal end of IMN, showing all possible degrees of deformation.

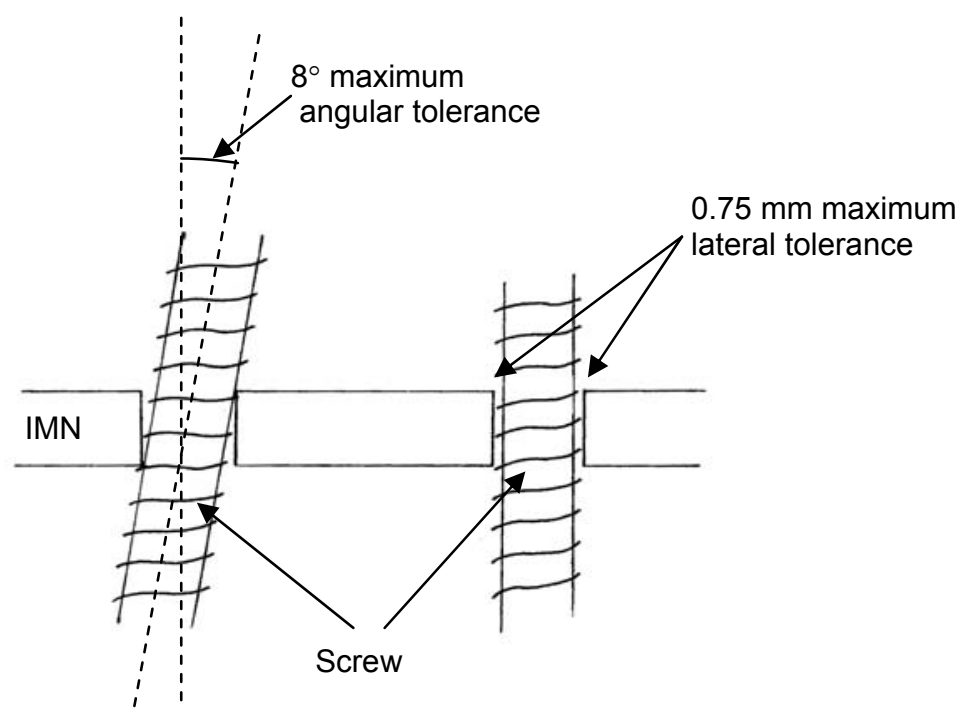

Figure 3) Maximum tolerances for screw insertion angle and translation.

If the drill bit is not within the tolerance constraints or misses the hole, a second attempt must be made. Unfortunately, once a hole is started, it is difficult to correct. In some cases, the bone may be too weak to accommodate another hole, and then bone grafting or other means of fracture fixation must be employed. Repairing a missed interlocking can involve more damage to the patient's bone and soft tissue, while both the surgeon and patient must also endure additional x-ray radiation. It is reported that femur 
fracture malpractice, in which IMN's are often used, ranks first on the list of most frequent and third on the list of most expensive lawsuits against orthopedic surgeons. ${ }^{2}$ For these reasons, many novel procedures and devices have been developed to assist the surgeon in distal locking of intramedullary nails correctly. These include mechanical guides, radiolucent targets, $\mathrm{x}$-ray dependant devices, c-arm mount devices, and magnetic targeting. Each of these devices will be presented, along with the original freehand targeting technique, still preferred by many surgeons.

Initially, surgeons used the freehand technique. This technique requires the surgeon to align the tip of the drill bit at a point on the surface of the bone just above the interlocking hole, under x-ray imaging. Correct alignment is indicated when the desired interlocking hole appears as a perfect circle under fluoroscopy, with the drill bit in the exact center. ${ }^{4}$ If alignment is lost, the drilling must be stopped and realigned using x-ray. The process of drill bit centering requires the soft tissue of the patient's extremity to be separated all the way to the bone so that the surgeon has sufficient room to maneuver. The desire to reduce damage of soft tissue during surgical procedures has led to the trend of percutaneous operating techniques. Percutaneous procedures are defined as inserting instruments and doing operations only through small skin incisions, reducing soft tissue damage. Overall, it has been realized that freehand alignment has many drawbacks such as excessive $x$-ray exposure, soft tissue damage, time consuming, and no real-time feedback of drill bit position.

Many aiming devices have been patented over the years. Some of these rely on the mechanical approach to distal hole location. ${ }^{4,8,12,16}$ The simplest of these mechanical devices uses an external arm with holes that correspond to the hole locations in the IMN. Once the IMN is implanted, the external guide arm is attached firmly to its proximal end, creating a solid link. In theory, the distal targeting holes will then be aligned with the distal interlocking screw holes of the IMN, ultimately allowing a straight shot for the drill to penetrate the bone and holes.

Problems with externally mounted jigs arise because they don't account for IMN deformation. In response, attempts have been made to correct for the inaccuracies of current mechanical targeting. Several devices exist which have an external drill guide arm with an extra reference pin attached to correct for bending of the nail. These devices correct for nail flexion in the sagittal plane only and do not account for deformation in the frontal plane and axial rotation of the nail, which have been proven to be significant for misalignment in previous studies. ${ }^{10,11}$ The device still requires $\mathrm{x}$-ray for alignment of the reference pin, which also adds soft tissue trauma, an extra hole in the bone, and additional surgery time. These studies indicate that an externally mounted, mechanical aiming device of this kind is not adequate for targeting the distal holes. ${ }^{10}$

While some devices aim to eliminate use of x-ray imaging all together, others have attempted to minimize its use and maximize the efficiency. There are a multitude of devices in this category. ${ }^{4,9,14,17,18,19}$ Generally, each employs some type of drill guide that is positioned under fluoroscopic imaging and then fixed in place when correctly aligned over the desired IMN interlocking hole. The dosage of radiation used with these devices is reduced compared to the amount used for freehand targeting. In addition, the surgical team is involved in many of these procedures each year from which their exposure compounds. The International Committee on Radiological Protection suggests limits on 
yearly occupational exposure. ${ }^{6,15}$ It is recommended that medical procedures adhere to the concept of "As low as reasonably achievable" when dealing with this matter because even the smallest dosage can cause abnormal cell growth. ${ }^{6}$ This statement is further support for development of distal targeting devices that are x-ray independent.

Additionally, x-ray devices must be employed in modern operating rooms equipped with c-arm fluoroscopy units and high powered computers. Consequently, they lose the advantage of portability and can not be used in primitive operating settings such as third world nations or temporary military hopsitals.

The desire to target accurately without fluoroscopic imaging has lead to the most recent attempts to use magnetic targeting. Devices have been developed that use external magnetic sensors to find the position of a flux field induced in the IMN by permanent or electromagnets. Some devices have even tried to magnetize the whole IMN and look for variations in the magnetic flux that occur around the interlocking holes. ${ }^{20}$ Others devices target on a magnet placed inside the IMN at the same position as the hole. ${ }^{3,5,7}$ The targeting device is usually attached to a drill sleeve to guide the bit for drilling.

Magnetic targeting has some significant advantages. Magnetic fields can penetrate the IMN and human tissue without being distorted or causing physiologic damage, unlike x-rays. Also, magnetic devices require little power, although some designs incorporate the use of computers to output positioning data. One notable drawback is that the majority of these targeting devices are manufactured to only work with specific nails, and are not adaptable to others. Additionally, devices that use electric current inside the body to create magnetic fields require stringent FDA approval because of their inherent danger. Although it is not yet perfected, magnetic distal targeting has shown the most promise, having the least drawbacks of all the x-ray independent techniques.

Design requirements for a targeting device should draw from the advantages of previous designs. The desired qualities describe a device with the following attributes.

- Requires minimal $\mathrm{x}$-ray exposure for targeting, not including the dosage that may be required for final assessment of correct interlocking.

- Low power, so it can run on a battery for use in primitive settings, such as third world countries and battlefield operating facilities.

- Simple, so that the surgeon does not require extensive training to operate the device.

- Accurate and repeatable, so that the holes may be drilled inside the allowable tolerances and without requiring complicated set up or calibration procedures.

- Percutaneous, to keep soft tissue damage and the external appearance of scarring to a minimum.

- The device should be adaptable to most nail designs.

- Real time targeting, providing feedback of position while aligning and during drilling. 


\section{Approach}

Using these design requirements, and building on what has been done successfully in the past, it is possible to develop a better distal interlocking screw targeting device and procedure. As mentioned, the magnetic targeting devices have shown great promise in providing optimal distal targeting independent of x-ray. Magnetic flux can penetrate human tissue and bone just as x-rays, but without the danger of radiation. In addition, the magnetic field sensors are used externally, so there is no soft tissue damage required for targeting, as in some other mechanical devices. Magnetic field sensors also have high resolution and are able to realize minute changes in their position within a field. For these reasons, magnetic field sensing has been adapted to the development of this IMN distal hole targeting design.

In order to align the drill bit with the axis of the desired interlocking hole, the surgeon must have feedback of positioning for rotation and translation in three dimensions. A coordinate axis was introduced in Figure 2, where it is assumed that the axis of the interlocking hole is $\mathrm{z}$, and the $\mathrm{x}-\mathrm{y}$ plane, normal to the face of the hole, is coplanar with the medial plane. This is the direction from which the surgeon will be locating and drilling the hole in the bone. The focus of this research is to use a permanent magnet and magnetic sensors to provide feedback for the surgeon to align the drill bit so that it is parallel to the axis of the hole for drilling.

This prototype targeting device will build on the existing magnetic distal screw insertion device developed by Dr. Durham. ${ }^{5}$ The aforementioned device uses a magnet placed inside the IMN at a point in front of the distal screw hole to be targeted. The magnet is inserted on a rod through the proximal opening in the hollow nail, while its insertion depth is fixed by a locking pin. Once the magnet is in front of one of the distal holes, a skin incision is marked using a magnetic compass to locate the position of the internal magnet which projects central flux lines parallel to the axis of the hole. After the skin and tissue are separated to allow working room, another magnet on a central pivot inside a tube is inserted down to the bone surface. These two magnets attract each other and align a guide wire which is then inserted. The magnets are removed and a cannulated drill bit is advanced over the guide wire which is now aligned directly with the axis of the hole. Finally the interlocking screw is inserted and the procedure is repeated for the more proximal hole.

This new prototype uses the existing magnet insertion techniques, but applies an electronic approach to the targeting problem. The targeting device developed by this research is used to locate a permanent magnet that will be locked in place offset from the hole to be drilled. The north pole of the magnet must face medially (along the z-axis) so that it projects a magnetic field having a central line of flux parallel to the axis of the interlocking hole. From outside the extremity in which the IMN is inserted, the targeting will be performed by an array of magnetic sensors held parallel to the medial plane. These sensors will be embedded in a targeting device handle, which will have a drill sleeve attached at its distal end. Acceptable drill sleeves have been developed previously that could be retrofitted to this design. The distance between the center of the drill sleeve and the center of the magnetic sensor array will be equivalent to the distance between the magnet and hole inside the IMN. The sensor array is aligned correctly over the internal magnet, therefore the drill sleeve will be aligned with the hole to be drilled. The surgeon 
can advance the drill bit through without hitting the magnet, while maintaining alignment feedback in real time. The locking mechanism can be adapted from the previous design. ${ }^{5}$ In the current design, a readout on the handle of the targeting device will have a "bullseye" of LED's which indicate position. The outside of the display will consist of a ring of red LED's, with one green LED in the center. The red LED's light to indicate which position the drill sleeve must move to have correct alignment. When aligned correctly, all red LED's will be off and the central green LED will be lit. The surgeon is then ready to drill the hole and insert the interlocking screws without aid of fluoroscopy or extraneous targeting systems. A schematic of the perceived device is presented in Figure 4.

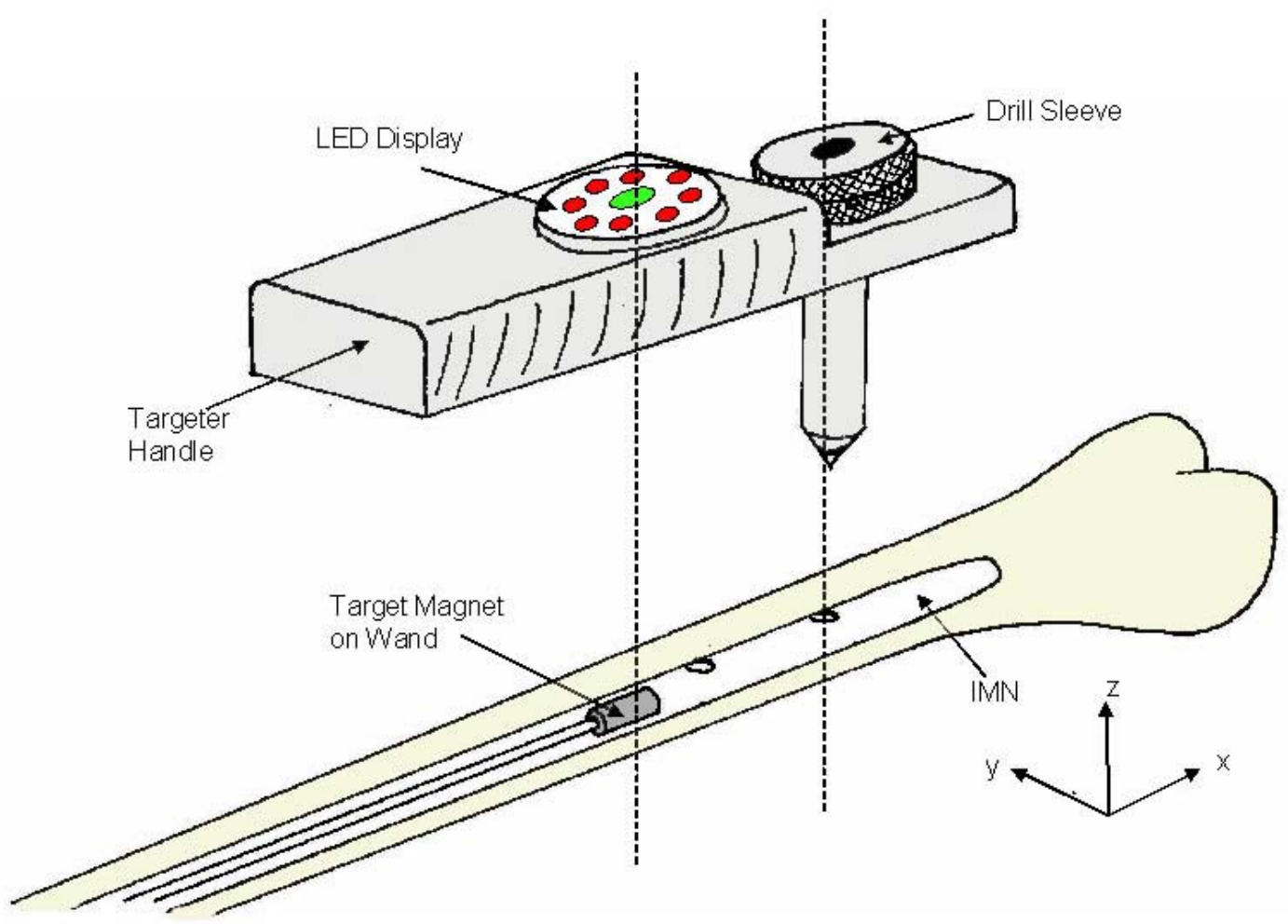

Figure 4) Perceived distal interlocking screw hole targeting device. The correct axis of alignment between the drill sleeve, hole, sensors, and target magnet are shown as the dotted lines. 


\section{Magnetic Field Targeting}

To implement magnetic field targeting, issues including the target magnet, sensors, and electronics are considered. Different options are discussed for each issue. Computer modeling of the magnetic field will be shown. This will be compared to experimental results from mapping the voltage about the magnet with the chosen sensors. The functional sensor configuration for targeting is developed. The sensor array output is then used to provide visual feedback of drill bit position for the surgeon. The target magnet and sensor array are shown as adapted to the process of locating and aligning a surgical drill and its bit parallel with the interlocking screw holes of the IMN. Conclusions, recommendations for improvements, and alternative applications are presented.

\section{a) Target Magnet Selection}

In order to align and advance the drill accurately, the surgeon must know where the drill is in relation to the axis of the hole. This requires a target magnet that provides targeting information to resolve all degrees of freedom. Therefore, the magnetic field from inside the nail must have a shape and polarity that affords unique targeting information in all possible planes. For targeting with this approach, the flux lines have a peak and a non-circular field shape about the axis of each plane so that the targeting sensors may be aligned. A non-circular, axisymmetric field was selected, allowing the sensors to distinguish rotation about the z-axis, while the peak flux lines perpendicular from the magnet indicate its exact center.

In verification of this design, Ansoft's Maxwell 3D magnetic modeling program was used to compare various magnet shapes and orientations. The shape and polarity that was found to afford the optimal field, was a cylindrical Neodymium Iron Boron ( $\mathrm{NdFeB}$ ) magnet that is polarized across its axis. Figure 5 shows flux density above the magnet, as well as orientation of the magnet polarity. It can be seen that there is a definitive peak that remains parallel to the z-axis regardless of distance, which is important because the targeter and corresponding drill bit must remain parallel to the hole at all depths. The field from this magnet must be detectable at a maximum distance typically encountered between the center of the IMN and the outside of the patient's limb, which is approximately 10 centimeters. The peak of the field is detectable at this distance, also seen in Figure 5. For the small percentage of large patients whom have an IMN place in an extremity of exceptional diameter, the surrounding tissue can be compressed to bring the distance below 10 centimeters. 


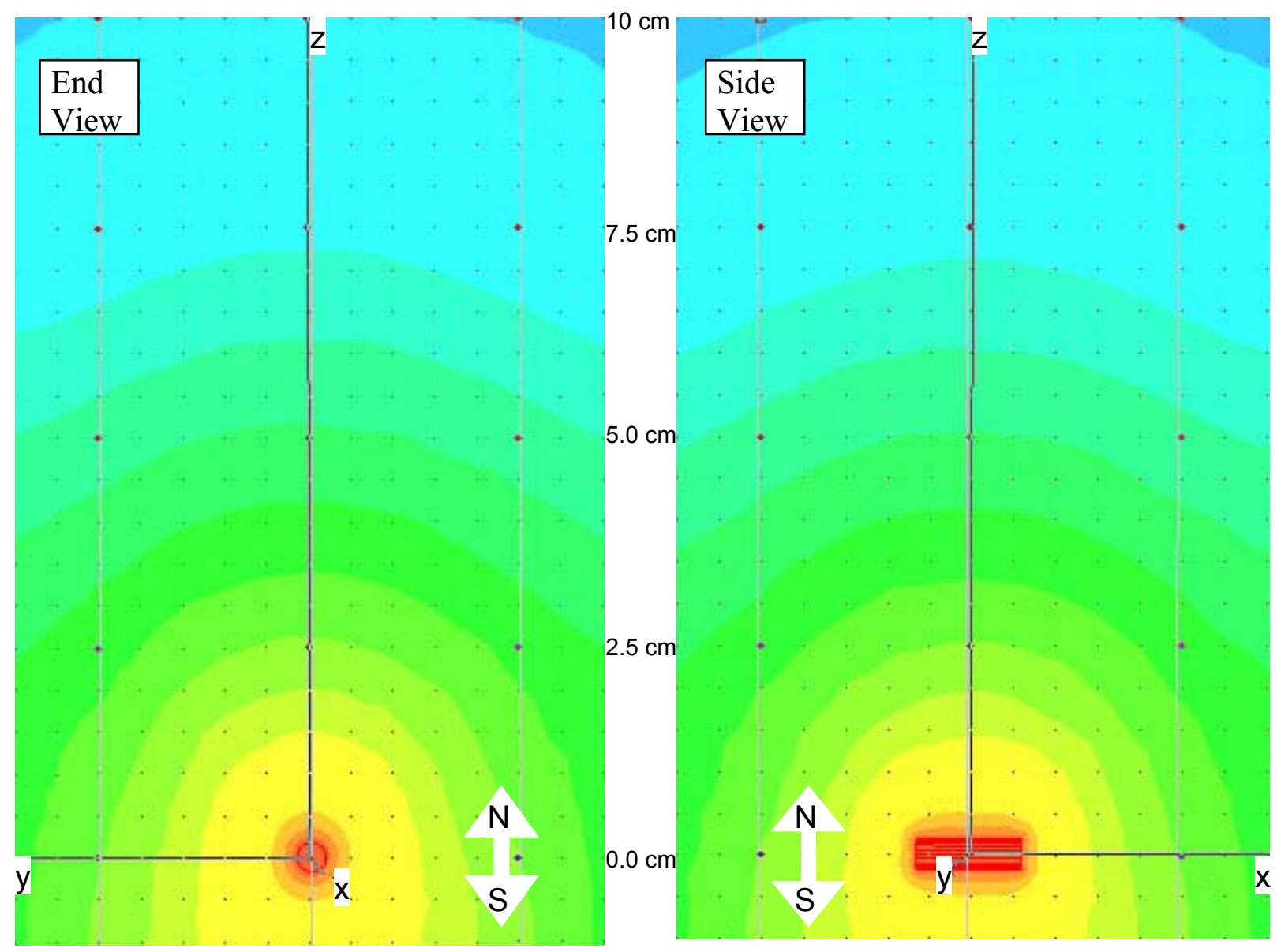

Figure 5) Plots of flux density in the $y-z$ and $x-z$ plane above the chosen cylindrical magnet. The peak is centered exactly over the magnet, parallel with the z-axis, and is detectable at the required targeting distance of $10 \mathrm{~cm}$.

The prototype targeting device will be aligned in the medial (x-y) plane, requiring a magnetic field that has an axisymmetric shape to resolve rotation about the z-axis. This elliptic field shape can be seen in Figure 6. The elliptic shape of the field, in the x-y plane, allows feedback of rotation and translation about the magnet, which is placed next to the IMN interlocking hole.

An experimental validation of this modeling of the axisymmetric field shape in the $x-y$ plane was performed. This was done by comparing the model's flus density plot of the x-y plane (Figure 6) to an actual mapping of the flux density recorded by a Gauss meter. The field of the magnet was mapped in $6 \mathrm{~mm}$ steps over a $10 \mathrm{~cm}$ by $10 \mathrm{~cm}$ area. To further support the claim that the chosen magnet projects the desired field shape to the required distance, the flux density mapping was performed at $+10 \mathrm{~cm}$ in the $z$-direction. It can be seen in Figure 7, that the experimentally recorded field shape is very similar to the model. 


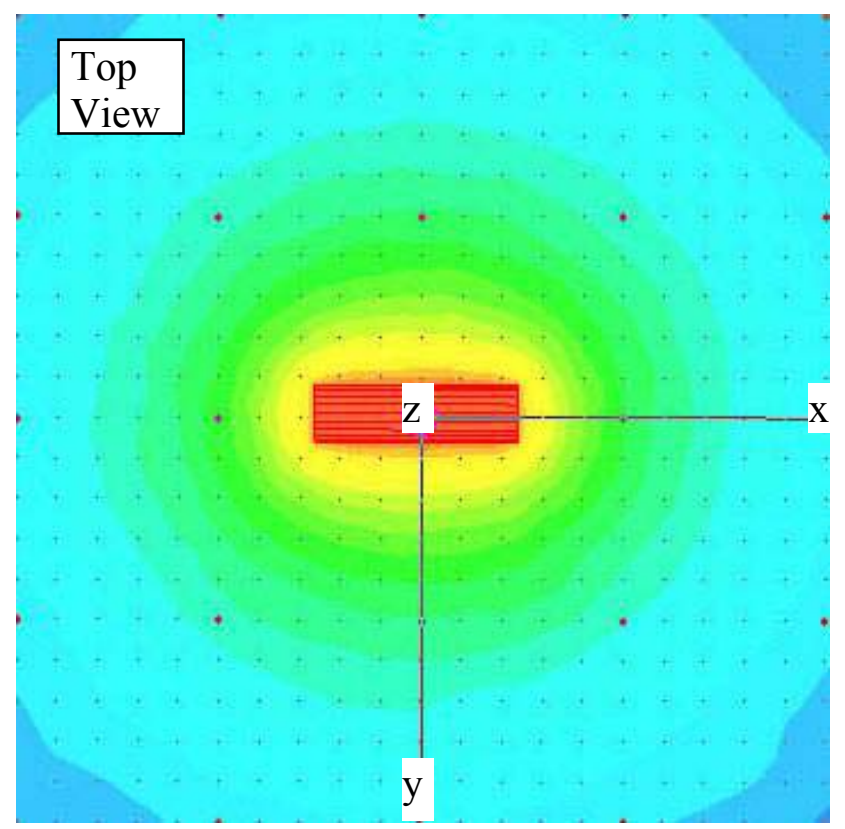

Figure 6) Plot of flux field around the magnet in the x-y plane. There exists a major and minor axis to the elliptic field, which can be differentiated by the targeting sensors to determine rotation about the z-axis. The north pole is facing in the positive $\mathrm{z}$ direction.

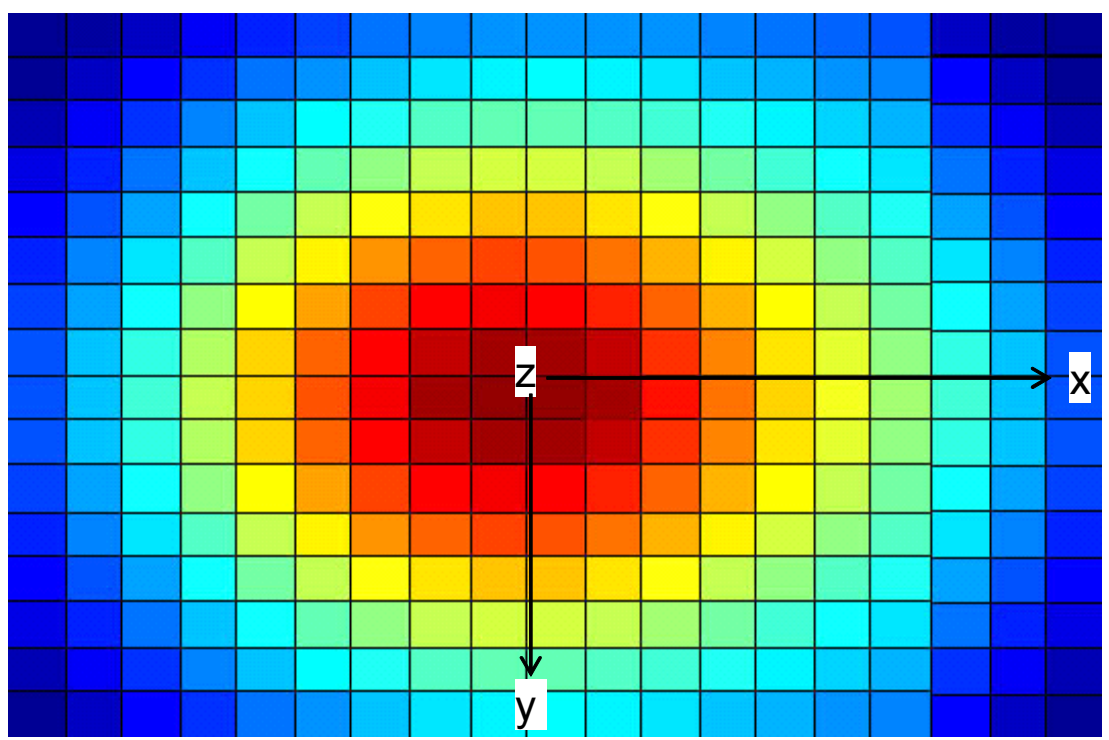

Figure 7) A portion of the mapping of flux density using a Gauss meter at $10 \mathrm{~cm}$ above the target magnet in the $+\mathrm{z}$-direction. Notice the axisymmetric field, as the model predicts in Figure 6. 
Another important consideration is the physical properties of the magnet itself. A single, cylindrical NdFeB magnet proves to be a good selection. They are already approved by the FDA for internal use. Compared to other materials used for magnets, $\mathrm{NdFeB}$ has good temperature stability, can be formed in many shapes while being magnetized as desired, and has high magnetic energy per volume. It is also relatively inexpensive and easy to manufacture.

\section{b) Targeting Sensors}

Magnetic field sensing for this type of targeting requires the measurement of both magnitude and direction of flux lines in the field of the target magnet. The sensor should have sufficient sensitivity and resolution to operate at a range of $10 \mathrm{~cm}$ from the target magnet. The field strength of the magnet at this distance will be below one Gauss, which is close to the value of Earth's magnetic field. Therefore, a sensor is needed that can sense very small changes in magnetic field. Sufficient resolution is gained by using a sensor that has a sensitive range of -2 to +2 Gauss. The field strength of the target magnet at the maximum $10 \mathrm{~cm}$ distance falls into this range.

Magnetoresistive (MR) sensors, produced by Philips, closely match the requirements for this application. These sensors can provide sensing feedback for small variations of magnetic fields at a distance of $10 \mathrm{~cm}$ from the target magnet. The sensitivity of the chosen sensors is 1.6 millivolts change per Gauss. The sensor readings can be nulled so that their outputs only represent changes in the flux lines emitted from the target magnet. This allows extraneous fields from other sources in the operating room to be ignored. Such extraneous sources could be the surgical drill, video monitors, lighting, and even the Earth's magnetic field.

The Philips MR sensors require only 120 milliwatts of power, which is appropriate for battery powered operation. The sensors can handle extreme environmental stress including high temperatures and chemicals. They can also be configured in an array, so that their collective outputs may be used for targeting.

\section{c) Targeting Array}

Each MR sensor produces a maximum output when flux lines are perpendicular to its sensitive side. This allows the angle and magnitude of the detected field to be known. In order to orient and align the targeting device in three dimensional space above the magnet, an elliptic array of sensors was developed. The array is configured so that the sensors are geometrically aligned with the flux lines of the target magnet. Each individual sensor is oriented perpendicular to the flux lines that project radially outward from the target magnet, while being centered about the peak flux lines emitted along the z-axis. A model of these flux lines in the $x-y$ plane can be seen in Figure 7, along with the optimal placement of the sensors in the plane. 


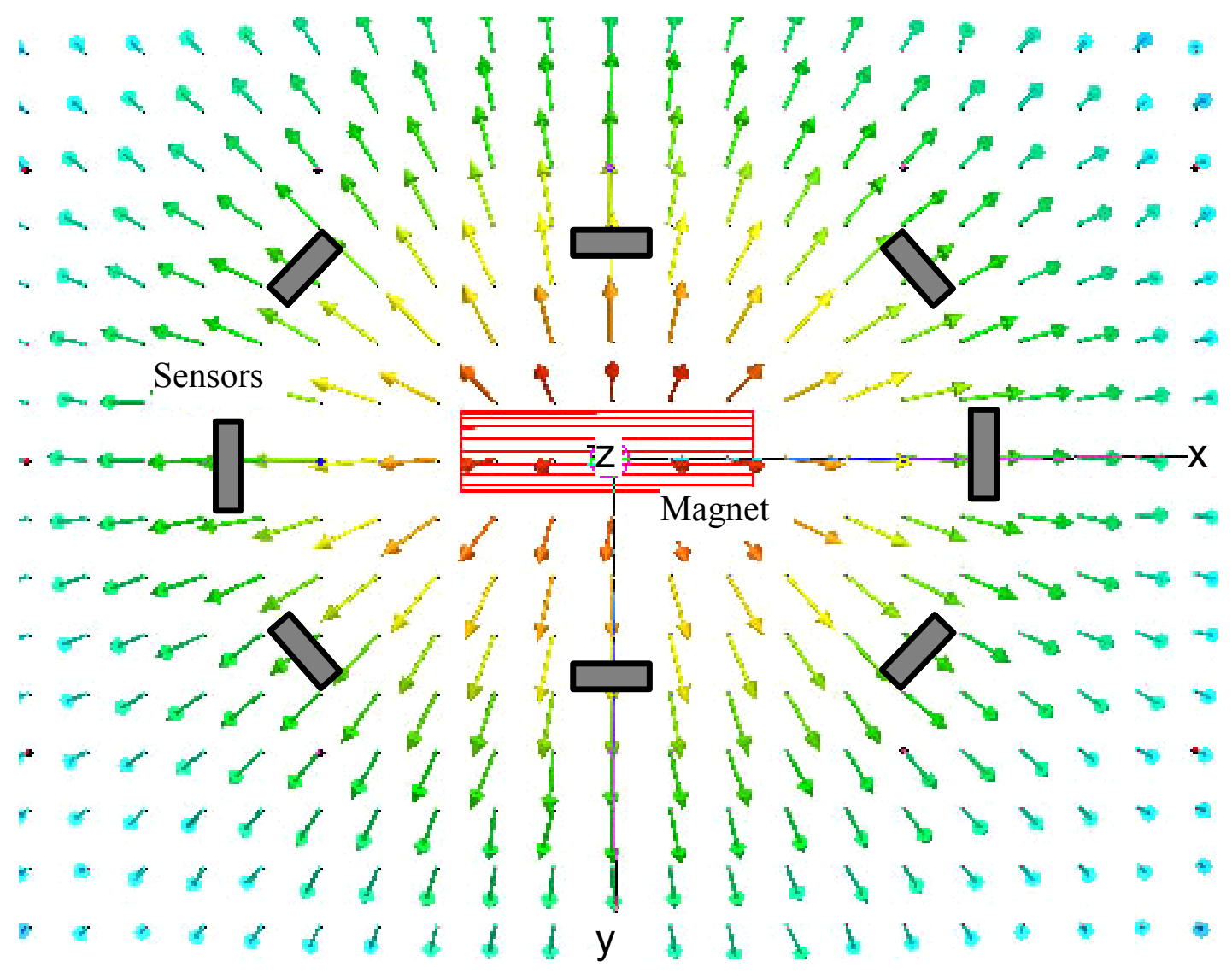

Figure 8) A Model of flux lines produced by the magnet. The elliptic array is shown, in which each sensor lies perpendicular to the flux field. This arrangement allows for equivalent voltage outputs from all the sensors when the array is centered in the flux field over the magnet.

This arrangement guarantees that each sensor in the array will be excited by the same magnitude and angle of flux when centered about the z-axis of the magnet, and will produce the exact same output voltage. The sensor array can move up and down in a plane perpendicular to the z-axis and retain the same feedback of position, because the field shape in that plane remains constant, it only becomes stronger as you get closer. When targeting, the voltage output of opposing sensors will be compared in order to determine their position and indicate which direction they need to be moved in the field relative to the peak.

\section{d) Positioning Feedback}

Using the sensor array output, continuous feedback is provided for the surgeon to center the drill above the interlocking hole in the medial plane. The positioning electronics are used to compare opposing sensor outputs and determine their location within the magnetic field, drive an LED display that visually indicates this position, and perform calibration of the sensors. 
The sensors in the targeting array are sensitive to small changes in magnetic field, making it possible to determine position in the field with a resolution of less than a millimeter in translation and less than one degree of rotation. Calibration is necessary because each sensor has an inherent offset at zero field due to manufacturing tolerances. Additionally, it is necessary to null any extraneous fields present in the operating room. Circuitry is provided that zeros the output of each sensor so that the array is ready for targeting. Calibration must be done with the sensors held away from any strong magnetic field, including that of the target magnet, so that the reading is not biased.

Once calibrated, it is possible to use the array of sensors to provide positioning data. The array is an ellipse of eight sensors, arranged with four sensor pairs, each opposing one another. Recall that the elliptical sensor array is aligned exactly with the elliptical pattern of flux intensity around the center of the target magnet. Refer to Figure 7 , showing optimal sensors placement in the magnetic field. With this in mind, it can be said that when the array is centered exactly over the target magnet in the medial plane, all sensors will have equal voltage outputs. Any deviation from exact center, in either rotation or translation, will cause an offset in opposing sensors pairs. By comparing voltage offset between opposing sensors in the array, it can be determined which direction in the field they must be moved to have equivalent outputs. A picture the sensor array board can be seen in Figure 9.

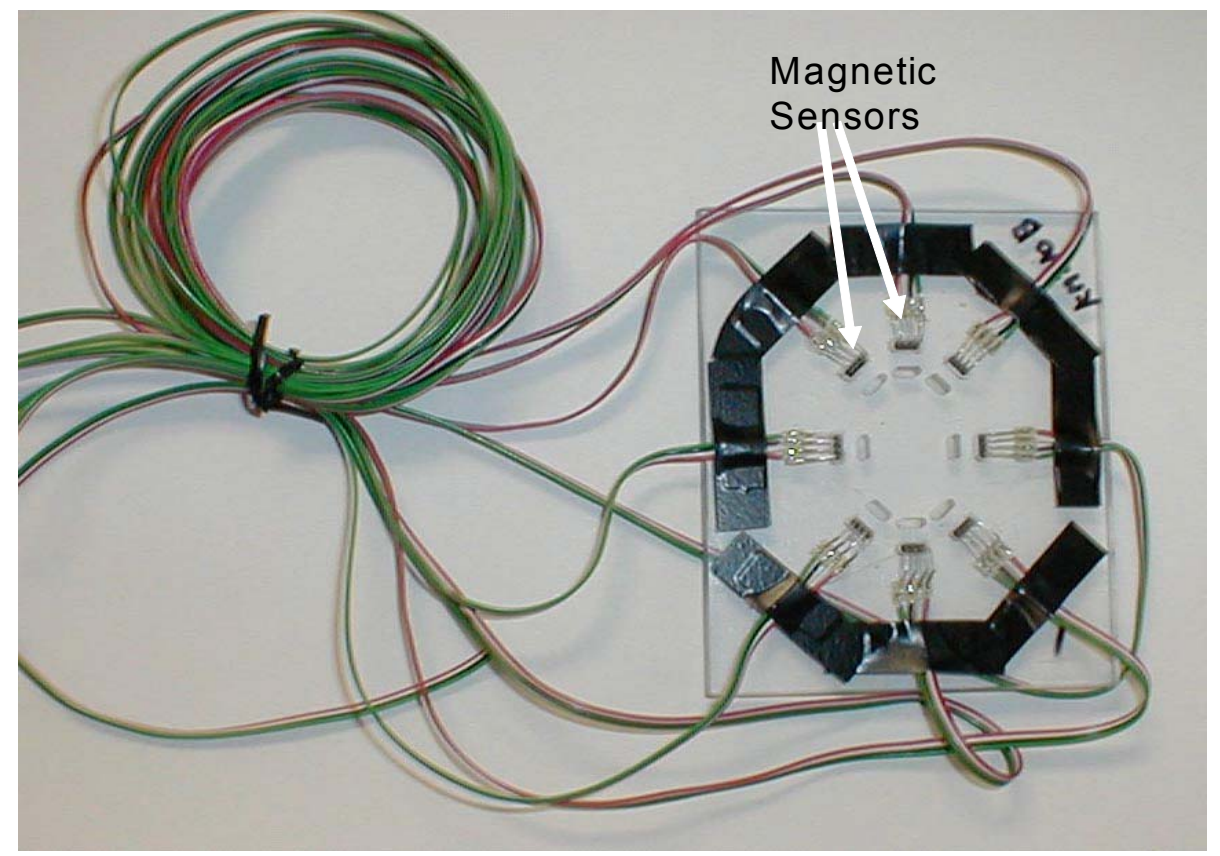

Figure 9) Arrangement of sensors fixed in a plastic housing to create an ellipse of opposing sensors pairs.

In order to convert these output voltages into a tangible indication of targeter position for the surgeon, a display must be created. The chosen display will be a "bullseye" of LED's, a ring of eight red lights around a central green light. Each LED will be tied to the corresponding sensor in the elliptic array. If a sensor pair has a voltage 
difference between them, it will be indicated on the LED display. An illuminated red LED means that the targeter must be moved in that direction to center itself. The position indicating LED's have a variable brightness that will decrease when the targeter is moving toward correct alignment. When all red LED's in the ring are off, the central green LED will light, indicating that the targeter is centered and ready for drilling. A picture of the LED display board can be seen in Figure 10. This visual feedback display can be mounted on a handle attached to a drill sleeve or even to the drill itself. Other feedback mechanisms could easily be incorporated if desired, such as tactile or audible indicators of position.

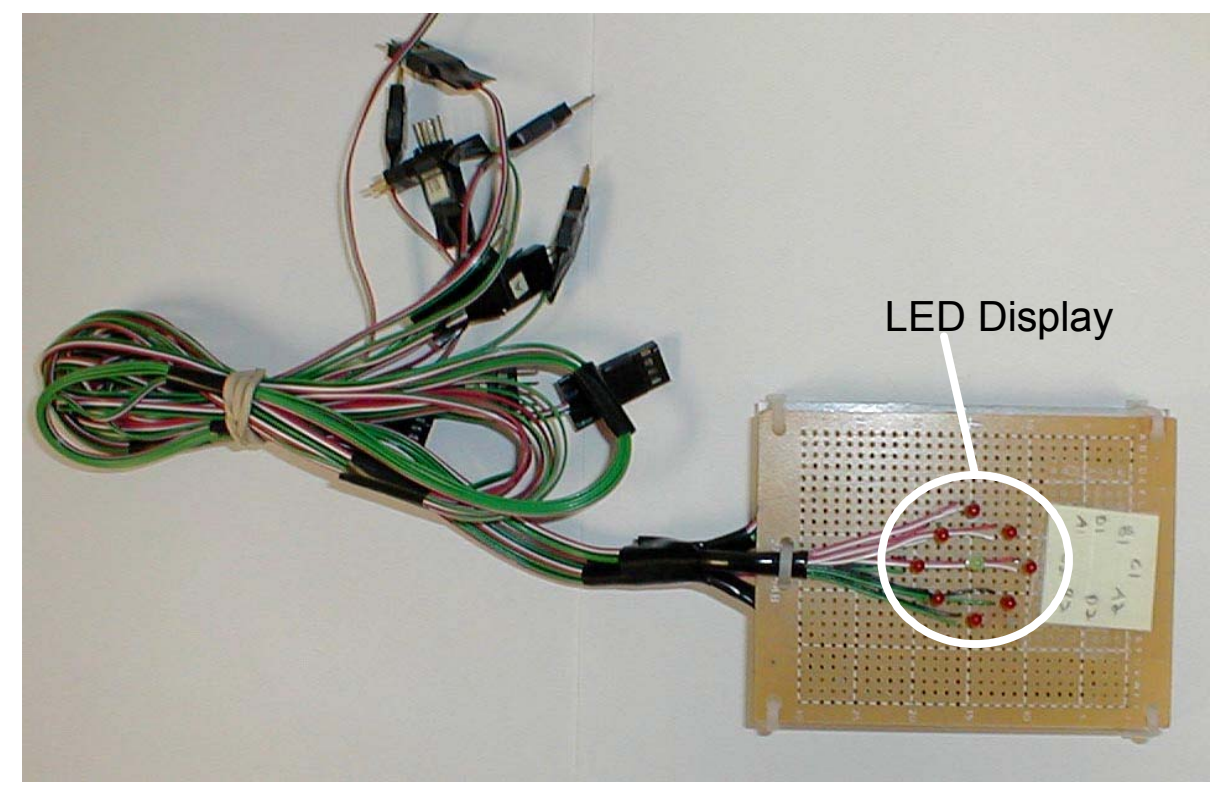

Figure 10) LED display arranged on the board in the form of a "bull's eye" for indicating correct alignment.

\section{Prototype}

A conceptual prototype which includes the target magnet, sensor array, visual display, and all necessary electronics has been developed. The prototype targeting device can be seen in Figure 11, without a handle or drill sleeve. Electronics are not shown. A schematic for the application of the prototype as a distal screw hole targeting device can be seen in Figure 4 and again in Figure 12, where a cut away of the embedded sensors are shown centered over the field of the target magnet. The prototype was developed and tested as a magnetic positioning device, able to locate itself relative to a target magnet. 


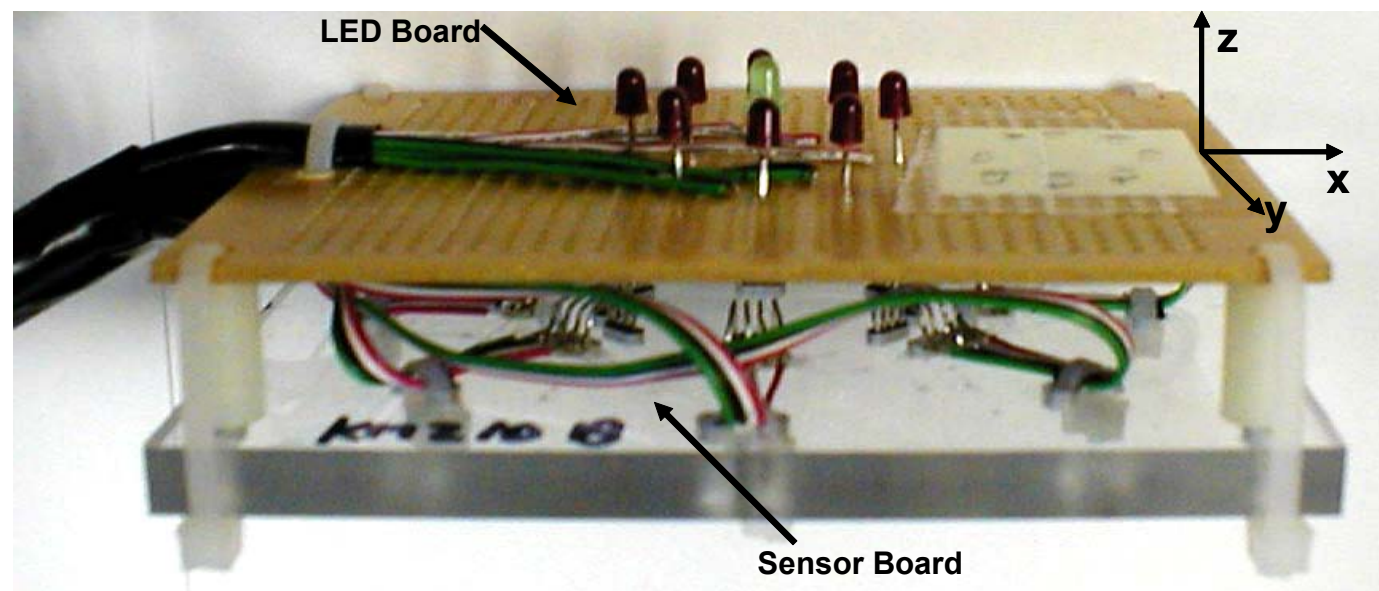

Figure 11) Prototype sensor array and LED display boards are shown connected and ready for targeting. The attached wires lead to the external positioning and calibration circuits.

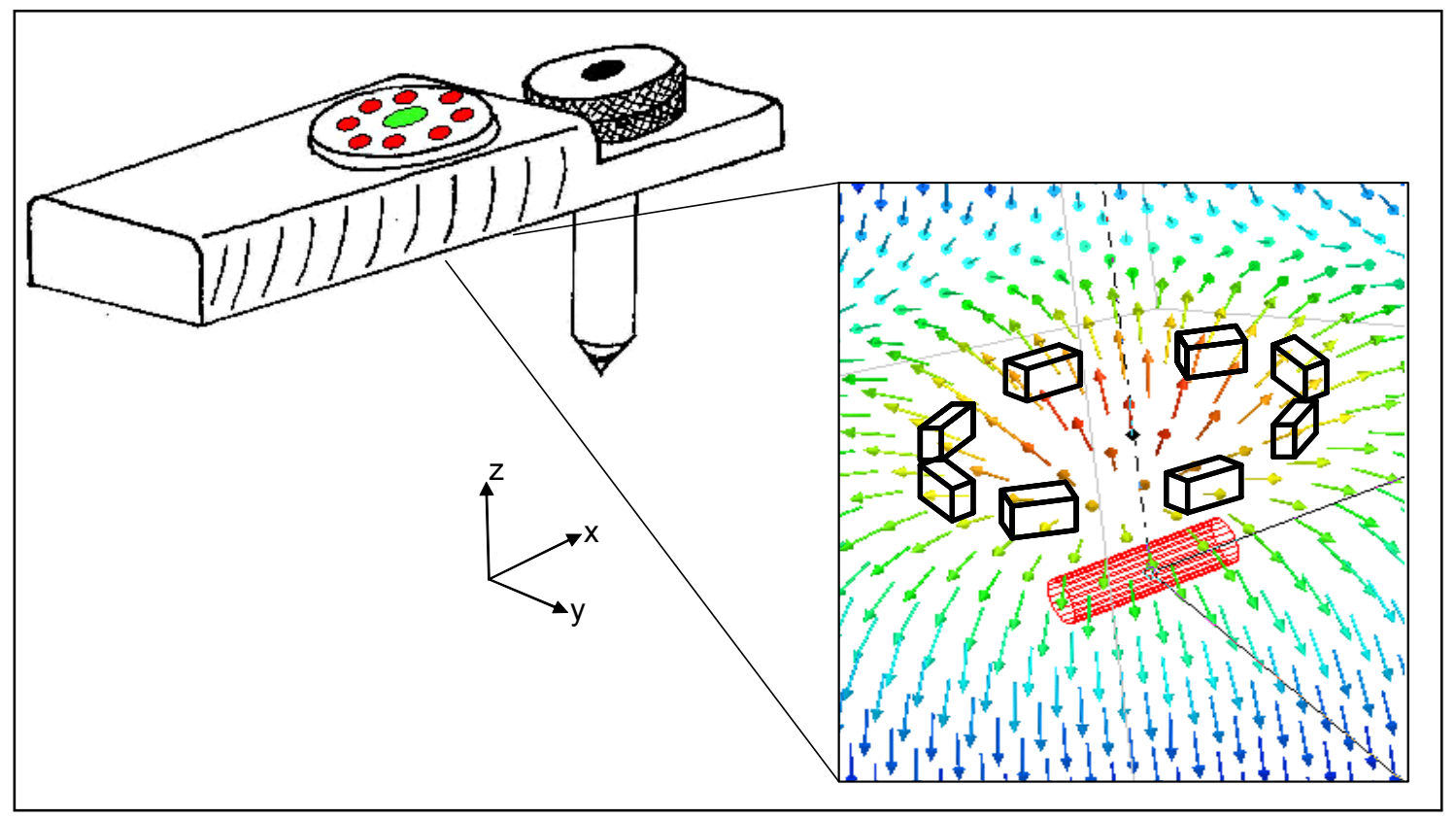

Figure 12) The sensors embedded in the handle are shown centered over the target magnet. 
The prototype includes a calibration circuit used to zero the sensors prior to targeting. This allows any extraneous fields and individual sensor offsets present in the output to be canceled. A target magnet is then located by continuous feedback from the LED display. In the chosen configuration, the LED display will indicate if the sensor array is not flat in the medial plane or off center from the magnet. The ring of LED's will be off and the central green LED illuminated when alignment of the drill bit is achieved. When applied to IMN distal interlocking screw hole location, feedback of position is available for alignment and also during drilling. The LED display can even be used to make sure the screw is driven properly. Each successive interlocking screw hole may be fixed in this manner, working back proximally from the end, by pulling back the magnet to correct alignment with the next hole. A schematic of the targeter, showing the centering of the sensor array over the magnet can be seen in Figure 11.

This prototype device has the ability to distinguish rotational misalignment of about one degree, which is indicated by the appropriate LED's on the display. Sensitivity to rotational misalignment was determined by holding the prototype in a test fixture and rotating the target magnet below at a distance of $10 \mathrm{~cm}$. Translational misalignment sensitivity was determined by placing the prototype in a test fixture and moving it in the $\mathrm{x}-\mathrm{y}$ plane at a distance of $10 \mathrm{~cm}$ above the target magnet. An offset in the targeting plane of less than a millimeter from center caused indication of misalignment on the LED's. The sensitivity of this device is completely adjustable in the case that the user wants a different feel to the targeter. A change in the positioning electronics will allow almost infinite adjustment of sensitivity, which could be integrated as a variable switch. Reduction of sensitivity may also be required if targeting on an extremity where the sensor and magnet gap is smaller. An example in distal IMN screw hole targeting would be that the tissue surrounding the femur is much thicker than around the tibia, which affects the sensing distance and creates the need for adjustable sensitivity so that the electronics are not overloaded when the sensors are used in a stronger field.

\section{Discussion}

Orthopedic surgeons have expressed that one of the more difficult parts of IMN surgery, is locating and drilling the interlocking screw holes. Distal interlocking is a critical part of IMN surgery, and is also liable to present complications. The devices created to aid surgeons in distal hole targeting have been only marginally successful, while adding their own complications to the procedure. A new distal interlocking screw hole targeting device has been developed in accordance with the specified design requirements. This prototype device features an array of magnetic sensors, a target magnet, and necessary electronics for targeting and calibration.

This device is able to resolve all degrees of freedom needed to accurately align the drill bit with the central axis of the interlocking hole, within the given tolerances. This device gives feedback of position in real time, so that alignment can be maintained during drilling. The prototype device achieves targeting without x-ray exposure. Although fluoroscopy may be employed to check proper screw interlocking, this device has the potential to eliminate $\mathrm{x}$-ray use during targeting. 
The application of this prototype allows for a percutaneous approach to interlocking screw hole targeting and drilling. Also, it can be used to locate the exact location of skin incisions needed above the interlocking holes for insertion of the drill bit. A visual positioning display was created to provide feedback of drill alignment during targeting. It is also possible to provide configure the prototype to provide audible and tactile feedback as well. The prototype includes a calibration circuit used to zero the sensors prior to targeting. This calibration can negate the effects of extraneous magnetic field present in the operating room.

This device also has additional benefits. The prototype's target magnet could be adaptable to any nail, providing the nail is hollow and non-ferrous. The cylindrical magnet shape, with a diameter of less than $4 \mathrm{~mm}$, allows the magnet to be placed lengthwise in the smallest, hollow IMN's used for bones such as the humorous or tibia. The device has low power requirements and can be powered by battery. The prototype can be incorporated with existing drill sleeves, IMN's, and magnet insertion wands, while only needing a handle to be fabricated to connect all the pieces.

\section{Conclusions}

The prototype device developed has the potential to improve orthopedic surgeons' ability to target and drill distal IMN interlocking screw holes. The device functions as a precision magnetic targeter which orients a sensor array with the axis of a target magnet, providing feedback of alignment. Further development and testing is being performed to take this prototype to a functional stage for use in the operating environment. The electronics must be miniaturized, and a sterilizable housing and drill sleeve created for the final device. Phantom bone, cadaveric, and clinical studies must be performed to further the advancement of this device. It is anticipated that orthopedic surgeons would welcome such a device that could improve the success rate and healing time after IMN surgery by reducing operating room time, $\mathrm{x}$-ray exposure, soft tissue trauma, and difficulty of interlocking. 


\section{References}

1) (1999) 20th Century Orthopaedics. The American Academy of Orthopaedic Surgeons 47.

2) (2001) Femur fracture care frequent cause of lawsuit. American Academy of Orthopaedic Surgeons 49.

3) Catamo,L., Rotini,R., Rocca,M., Giardino,R., and Fontanesi,G. (1998) Distal centering in locked intramedullary osteosynthesis of the femur: use of a magnetresistant probe. Chir Organi Mov 83, 375-379.

4) Court-Brown (1991) An Atlas of Closed Nailing of the Tibia and Femur. Springer-Verlag, New York.

5) Durham,A.A. and Crickenberger,D.P. (1998) Magnetic Distal Targeting for Modular Intramedullary Nails. Techniques in Orthopaedics 13, 71-78.

6) Evans,R.J., Cusack,S., and Parke,T. (1992) Exposure of the hands to ionizing radiation in the resuscitation room of an accident \& emergency department. Arch Emerg.Med. 9, 220-224.

7) Holistien,S. and Ruth,J.T. (1996) Electromagnetic distal targeting for the placement of transverse interlocking screws. Osteosynthese International 3, 196203.

8) Karachalios,T., Babis,G., Tsarouchas,J., Sapkas,G., and Pantazopoulos,T. (2000) The clinical performance of a small diameter tibial nailing system with a mechanical distal aiming device. International Journal of the Care of the Injured 31, 451-459.

9) Kelley,S.S., Bonar,S., Hussamy,O.D., and Morrison,J.A. (1995) A simple technique for insertion of distal screws into interlocking nails. J.Orthop.Trauma 9, 227-230.

10) Krettek,C., Konemann,B., Mannss,J., Schandelmaier,P., Schmidt,U., and Tscherne,H. (1996) [Analysis of implantation-induced nail deformation and roentgen morphometric studies as the principle for an aiming device for distal interlocking nailing without roentgen image intensification]. Unfallchirurg 99, 671-678.

11) Krettek,C., Konemann,B., Miclau,T., Schlandermaier,P., and Blauth,M. (1997) In vitro and in vivo radiomorphic analyses of distal screw hole position of the solid tibial nail following insertion. Clinical Biomechanics 12, 198-200. 
12) Krettek,C., Konemann,B., Miclau,T., Kolbli,R., Machreich,T., Kromm,A., and Tscherne,H. (1998) A new mechanical aiming device for the placement of distal interlocking screws in femoral nails. Arch Orthop.Trauma Surg 117, 147-152.

13) Krettek,C., Mannss,J., Miclau,T., Schandelmaier,P., Linnemann,I., and Tscherne,H. (1998) Deformation of femoral nails with intramedullary insertion. J.Orthop.Res. 16, 572-575.

14) Mahaisavariya,B., Laupattarakasem,W., and Kosuwon,W. (1992) An aiming device for distal locking in closed locked femoral nailing. Injury 23, 143-144.

15) Noordeen,M.H., Shergill,N., Twyman,R.S., Cobb,J.P., and Briggs,T. (1993) Hazard of ionizing radiation to trauma surgeons: reducing the risk. Injury 24, 562564.

16) Pennig,D., Oppenheim,W., Faccioli,G., and Rossi,S. (1997) Intramedullary locked nailing of femur and tibia: Insertion of distal locking screws without image intensifier. Injury 28, 323-326.

17) Slomczykowski,M.A., Hofstetter,R., Sati,M., Krettek,C., and Nolte,L.P. (2001) Novel computer-assisted fluoroscopy system for intraoperative guidance: feasibility study for distal locking of femoral nails. J.Orthop.Trauma 15, 122-131.

18) Tyropoulos,S. and Garnavos,C. (2001) A new distal targeting device for closed interlocking nailing. International Journal of the Care of the Injured 32, 732-735.

19) Viant,W.J., Phillips,R., Griffiths,J.G., Ozanian,T.O., Mohsen,A.M., Cain,T.J., Karpinski,M.R., and Sherman,K.P. (1997) A computer assisted orthopaedic surgical system for distal locking of intramedullary nails. Proc.Inst.Mech.Eng [H.] 211, 293-300.

20) Zacheja,J., Bach,T., and Clasbrummel,B. (2000) Application of Microsensors for Minimally Invasive Vascular Flow Measurements and Fracture Repair Systems., Hanover, Germany. 


\section{Vita}

David C. Szakelyhidi Jr. was born on July $28^{\text {th }}, 1978$ in Butler, Pennsylvania. After graduating from Seneca Valley High School in Harmony, Pennsylvania, he studied mechanical engineering at West Virginia University in Morgantown, West Virginia, and graduated with a Bachelor of Science degree in 2000. He continued his education in 2000 in the Mechanical Engineering Department at Virginia Tech. He received his Master of Science degree in Mechanical Engineering, with an option of Biomedical Engineering, in May of 2002. Following graduation he will work as a research associate at Virginia Tech, as an extension of his ongoing biomedical research. Eventually he has a goal of employment as researcher for a biomedical engineering company. 